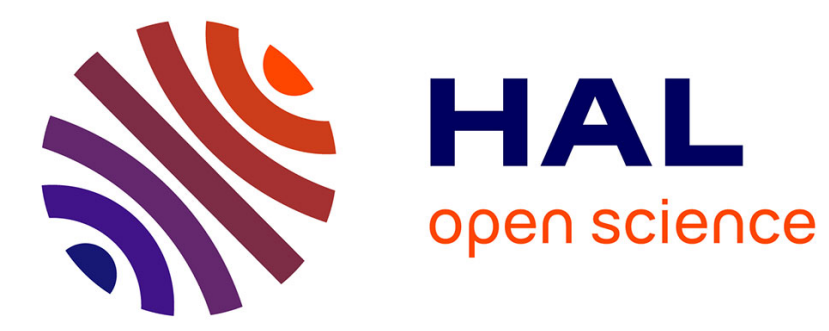

\title{
Event-triggered observer design for linear systems subject to delayed and sampled output
}

Chengcheng Song, Haoping Wang, Yang Tian, Gang Zheng, Alexandre Seuret

\section{To cite this version:}

Chengcheng Song, Haoping Wang, Yang Tian, Gang Zheng, Alexandre Seuret. Event-triggered observer design for linear systems subject to delayed and sampled output. International Journal of Control, 2022, 95 (3), pp.581-588. 10.1080/00207179.2020.1805127 . hal-02941641

\section{HAL Id: hal-02941641 \\ https://hal.laas.fr/hal-02941641}

Submitted on 25 Nov 2020

HAL is a multi-disciplinary open access archive for the deposit and dissemination of scientific research documents, whether they are published or not. The documents may come from teaching and research institutions in France or abroad, or from public or private research centers.
L'archive ouverte pluridisciplinaire HAL, est destinée au dépôt et à la diffusion de documents scientifiques de niveau recherche, publiés ou non, émanant des établissements d'enseignement et de recherche français ou étrangers, des laboratoires publics ou privés. 


\title{
ARTICLE TEMPLATE
}

\section{Event-triggered observer design for linear systems subject to delayed and sampled output}

Chengcheng Song ${ }^{\mathrm{a}}$, Haoping Wang ${ }^{\mathrm{a}}$, Yang Tian ${ }^{\mathrm{a}}$, Gang Zheng ${ }^{\mathrm{b}}$, and Alexandre Seuret $^{\mathrm{c}}$

${ }^{a}$ The Sino-French International Joint Laboratory of Automatic Control and Signal Processing, School of Automation, Nanjing University of Science \& Technology, Nanjing 210094, China; ${ }^{\mathrm{b} T h e}$ Inria Lille, France; ' LAAS-CNRS, Université de Toulouse, Toulouse, France;

\section{ARTICLE HISTORY}

Compiled February 28, 2020

\begin{abstract}
In this paper, we investigate the observation and stabilization problems for a class of linear time-invariant systems, subject to unknown state, and network constraints, including time-delays and event-triggered sampling. A new type of event-triggered mechanism is proposed based on an appropriate storage function, which is chosen larger than the derivative of Lyapunov function. Thus, the convergence of the observer system is guaranteed by the negativity of this appropriate storage function. With the utilization of LMI techniques and the designed novel event-triggered mechanism, only a slight over-estimation exists in the process of obtaining stability conditions, leading to reduced conservatism. The effectiveness of this novel event-triggered mechanism is proven through a comparison with a classical event-triggered and also periodic time-triggered mechanisms, and through simulations of a mobile cart system.
\end{abstract}

\section{KEYWORDS}

Observation; stabilization; event-triggered sampling; time-delays; LMI; reduced conservatism.

\section{Introduction}

Event-triggered mechanism is an important and effective technique for aperiodic networked transmissions when communication and energy resources are limited, see (Chen \& Li, 2018; Heemels, Sandee, \& Van Den Bosch, 2008; Hetel et al., 2017; D. Zhang, Shi, Wang, \& Yu, 2017; X. M. Zhang, Han, \& Zhang, 2017) for introductions to the topic. The interests of considering an event-triggered condition is to decide whether to transmit the data or not. The event-triggered condition usually contains some known system's parameters or real-time information of systems, which allow to determine whether it is necessary to transmit data between components.

State observation (or estimation) is one of the central problem in system theory. Compared to event-triggered control problems, the event-triggered transmission 
applied to observers of networked systems has been less studied in the literature, to the best of our knowledge. Such as studies for linear continuous systems (Tanwani, Prieur, \& Fiacchini, 2016), and for linear discrete systems (Dong, Wang, Alsaadi, \& Ahmad, 2015; Han et al., 2015; J. Huang, Shi, \& Chen, 2017; Leong, Dey, \& Quevedo, 2017; Molin \& Hirche, 2017; Molin, Sandberg, \& Johansson, 2016; Shi, Chen, \& Ling, 2014; Z. H. Zhang \& Yang, 2017). However, these above literatures about event-triggered observers ignore the presence of delays, which actually will be considered in this paper. Besides, concerning the event-triggered observers, there are also studies based on nonlinear systems, such as for continuous nonlinear systems (Etienne \& Gennaro, 2016; Etienne, Gennaro, \& Barbot, 2017; Y. Huang, Wang, Shi, \& Shi, 2018; Li, Wang, Ma, \& Liu, 2019; Zou, Wang, Gao, \& Liu, 2017), and for discrete nonlinear systems ( $\mathrm{Chu} \& \mathrm{Li}, 2018$; Zheng \& Fang, 2016). For these studies of event-triggered observer systems, most of them use the classical event-triggered condition, which is based on the difference between current value and the last transmitted value, such as in (Chu \& Li, 2018; Dong et al., 2015; Etienne \& Gennaro, 2016; Etienne et al., 2017; Y. Huang et al., 2018; Li et al., 2019; Tanwani et al., 2016; Zheng \& Fang, 2016; Zou et al., 2017). The difference between current value and the last transmitted value is also called sampling error, see (Y. Huang et al., 2018). For this classical event-triggered condition, the output data is transmitted from the sensor to the observer through a network if the sampling error exceeds a designed bound. This classical kind of event-triggered mechanisms potentially mean that, if the data changes fast, then more transmissions are triggered, conversely, if it changes slowly, then less transmissions are triggered. It's better for getting more approximate dynamics of transmitted data, which is finally for getting a better estimation performance. From this point of view, designing a new event-triggered condition which is directly related to estimation performance, may bring less conservatism.

Thus, in this paper, the event-triggered condition is based on an appropriate storage function, which is larger than the derivative of a Lyapunov function, so that, if this particular storage function is guaranteed to be non-positive, then the derivative of Lyapunov function is negative. This storage function actually bounds the derivative of a Lyapunov function. So, in this novel event-triggered mechanism, if this appropriate storage function is predicted to be positive, then transmission will be triggered to correct it to be non-positive, otherwise the transmission will not be triggered. This new kind of event-triggered mechanisms is potentially committed to guarantee the convergence of the system since it guarantees the decrease of a Lyapunov function. Actually, this conception has already attracted researchers attention (Seuret, Prieur, Tarbouriech, \& Zaccarian, 2016; Tarbouriech, Seuret, Silva, \& Sbarbaro, 2016). However, paper (Seuret et al., 2016) studies a static state-feedback event-triggered controller with known state information, and paper (Tarbouriech et al., 2016) proposes an observer-based event-triggered controller in which the observer in sensor side uses the continuous output data, while in this paper the state information is unknown and an event-triggered observer is proposed far away from sensor side, only using aperiodic (event-triggered) sampled output. Besides, both of the studies (Seuret et al., 2016; Tarbouriech et al., 2016) ignore the network-induced time-delay when it is considered in this paper.

Comparing these two types of event-triggered conditions, including the classical error-based one and the new proposed one, their basic thinking is totally different. For 
the classical kind of event-triggered condition, the idea that more transmissions for fast-changing data and less transmissions for slow-changing data, is actually devoted to recovering the dynamics of the data. However, for the new proposed event-triggered condition in this paper, as mentioned above, it is committed to guarantee the decrease of the Lyapunov function, further guaranteeing the convergence of the system, which is directly related to estimation performance, further may bring less conservatism. In this paper, this new proposed method will be named as storage function based event-triggered mechanism.

Normally, some essential over-estimations exist when deriving stability conditions of the observer, for example in (Ahmed-Ali, Assche, Massieu, \& Dorleans, 2013) Young's inequalities are used, and in (Ahmed-Ali et al., 2013; Shoukry \& Tabuada, 2016) norm inequalities and some matrix inequalities are used. As we known, over-estimations lead to more conservative stability conditions. In this paper, with the utilization of LMI techniques and the designed novel event-triggered mechanism, a Wirtinger-based inequality from (Seuret \& Gouaisbaut, 2013) is used to derive efficient stability conditions, which leads to a slight over-estimation, and consequently, less conservative stability conditions, compared to Jensen inequality.

Thus, the contributions of this paper are as follows: (1) Based on aperiodic sampled and delayed output, an event-triggered state observer is proposed for a class of linear systems; (2)Differently from usual classical event-triggered schemes, a new type of event-triggered condition is designed which is more direct and less conservative; (3) Only a slight over-estimation exists in the process of obtaining stability conditions which leads to less conservative stability conditions.

\section{Prepiminaries}

In this paper, the symbol $\mathbb{R}$ denotes the set of real numbers. The symbol $\mathbb{N}$ denote the set of natural numbers. For $p, q \in \mathbb{N}, \mathbb{R}^{p \times q}$ stands for the set of real matrices of dimension $p \times q$. For $n \in \mathbb{N}, I_{n} \in \mathbb{R}^{n \times n}$ is the identity matrix of dimension $n \times n$. For $p, q, m, n \in \mathbb{N}$, if $F \subset \mathbb{R}^{p \times q}$ and $G \subset \mathbb{R}^{m \times n}, \mathcal{C}(F, G)$ denotes the space of all continuous functions mapping $F$ to $G$. For any vector or matrix $A$ of appropriate dimension, $A^{\prime}$ represents the transpose of matrix/vector $A . t_{0}$ represents the initial time. If $A \in \mathbb{R}^{n \times n}, H_{e}(A)$ is a simplified symbol of matrix $A+A^{\prime}$ in the sequel.

Also, the Wirtinger-based inequality in Lemma 2.1 will be used shown in the sequel:

Lemma 2.1. For any matrix $R>0$, the following inequality holds for any continuously differentiable function $\omega$ in $[a, b] \rightarrow \mathbb{R}^{n}$ :

$$
\int_{a}^{b} \dot{\omega}^{\prime}(s) R \dot{\omega}(s) d s \geq \frac{1}{b-a}(\omega(b)-\omega(a))^{\prime} R(\omega(b)-\omega(a))+\frac{3}{b-a} \Omega^{\prime} R \Omega
$$

where $\Omega=\omega(b)+\omega(a)-\frac{2}{b-a} \int_{a}^{b} \omega(s) d s$

The proof is omitted and can be found in (Seuret \& Gouaisbaut, 2013). 


\section{Problem formulation}

Consider the following continuous-time plant:

$$
\left\{\begin{array}{l}
\dot{x}(t)=A x(t)+B(u(t)), \quad t \geq t_{0}, x\left(t_{0}\right)=x_{0} \\
y(t)=C x(t)
\end{array}\right.
$$

where $x(t) \in \mathbb{R}^{n}, u(t) \in \mathbb{R}^{m}$ and $y(t) \in \mathbb{R}^{p}$ are the state, the input and the output of the plant, respectively, with $n, m, p \in \mathbb{N}$. Matrices $A \in \mathbb{R}^{n \times n}, B \in \mathcal{C}\left(\mathbb{R}^{m}, \mathbb{R}^{n}\right)$ and $C \in \mathbb{R}^{p \times n}$ are known. The pair $(A, C)$ are assumed to be detectable.

In this paper, the interest is to design an observer for plant (2), when its output is aperiodically sampled and contains a fixed time-delay, which is denoted by $\tau \in \mathbb{R}$ satisfying $\tau>0$. The sampled and delayed output is denoted by $z(t)=y\left(t_{k}-\tau\right)$ when $t \in\left[t_{k}, t_{k+1}\right)$. Define $\bar{t}_{k} \in \mathbb{R}$ satisfying $\bar{t}_{k}=t_{k}-\tau$, thus $\left\{\bar{t}_{k} \mid k \in \mathbb{N}\right\}$ is the set of aperiodic sampling instants, which are decided by the event-triggered mechanism, which will be designed later in the Section 5.1.

\section{Event-triggered observer design}

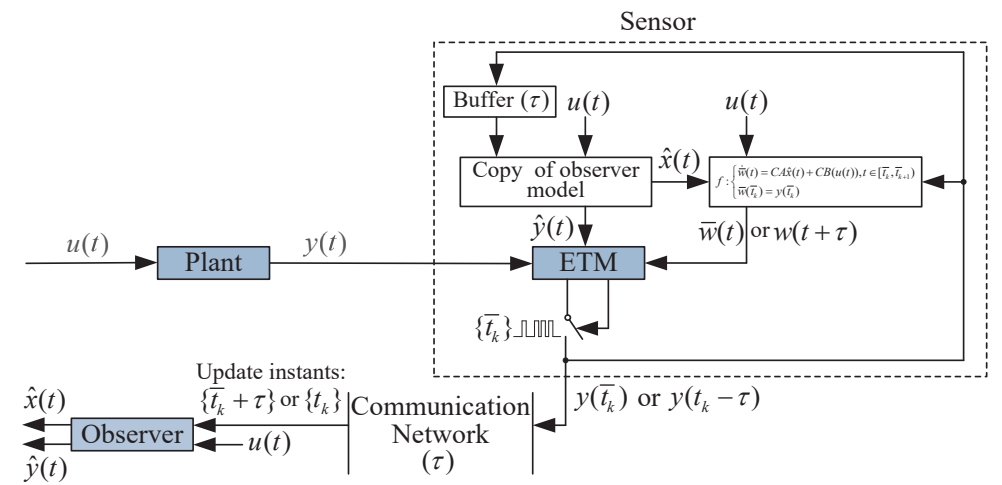

Figure 1. Event-triggered observer system (ETM: event-triggered mechanism)

The framework of event-triggered observer system is shown in Figure 1, and it includes a plant, a sensor with an event trigger rule, a communication network with time delays, and an observer far away from sensor. With the event trigger rule, the sensor send the aperiodically sampled output to the observer through the communication network which contains the time delays. Based on the framework shown in Figure 1, an event-triggered observer is designed for plant (2) as follow. It contains two main ingredients: 
- A classical Luenberger observer, whose input is a vector $w(t)$, to be designed:

$$
\left\{\begin{array}{l}
\dot{\hat{x}}(t)=A \hat{x}(t)+B(u)-L(C \hat{x}(t-\tau)-w(t)), \quad \forall t \geq 0 \\
\hat{y}(t)=C \hat{x}(t), \quad \forall t \geq 0
\end{array}\right.
$$

- A continuous estimation of the sampled and delayed output: (reset predictor)

$$
\left\{\begin{array}{l}
\dot{w}(t)=C A \hat{x}(t-\tau)+C B(u(t-\tau)), \quad t \in\left[t_{k}, t_{k+1}\right), \forall k \geq 0 \\
w(t)=y(t-\tau), \quad t=t_{k}, \forall k \geq 0
\end{array}\right.
$$

Indeed the variable $\hat{x}(t)$ is built to estimate $x(t)$, matrix $L$ is the observer gain of appropriate dimension, which will be designed by setting the poles of $A-L C$ at negative axis, $\hat{x}_{0}$ denotes the initial condition of the observer, $\hat{y}(t)$ is the estimate of the current output, and $w(t)$ is the state of reset predictor (4), which is updated by the real sampled and delayed output of the system. An event-triggered mechanism will be designed later to decide the sampling instants $\left\{\bar{t}_{k}=t_{k}-\tau \mid k \in \mathbb{N}\right\}$.

Let us define the state observation error as $e=\hat{x}-x$. Equations (2) and (3) yields the following dynamical equation:

$$
\begin{aligned}
\dot{e}(t) & =A e(t)-L(C \hat{x}(t-\tau)-w(t)) \\
& =A e(t)-L\left(e_{y}(t-\tau)-e_{w}(t)\right)
\end{aligned}
$$

where $e_{w}(t)=w(t)-y(t-\tau)$ is error of reset predictor and $e_{y}(t)=C e(t)$ is the measurement error.

\section{Main results}

\subsection{Design of the event-triggered mechanism}

The event-triggered mechanism is designed as follows:

$$
\bar{t}_{k+1}=\min \left\{t>\bar{t}_{k}, \text { s.t. }\left[\begin{array}{c}
C e(t) \\
e_{w}(t+\tau)
\end{array}\right]^{\prime} F\left[\begin{array}{c}
C e(t) \\
e_{w}(t+\tau)
\end{array}\right]>0\right\}
$$

where matrix $F=\left[\begin{array}{ll}F_{11} & F_{12} \\ F_{21} & F_{22}\end{array}\right]$ with $F_{11}, F_{12}, F_{21}, F_{22} \in \mathbb{R}^{p \times p}$ is a parameter to be designed satisfying

$$
F_{11}<0
$$

Remark 1. (Feasibility of event-triggered mechanism (6)) The event-triggered condition actually uses the information of observer, which is realized by setting a copy of 
observer model at sensor side, so that the variables in observer can be seen as available when designing event-triggered mechanism, and the observer model can be replicated to the sensor side since all information of observer is actually known for sensor side. Besides, in the event-triggered mechanism (6), a future value $e_{w}(t+\tau)$ is used, which actually can be realized: it's easy to get $e_{w}(t+\tau)=\bar{w}(t)-y(t)$ with $\bar{w}(t) \triangleq w(t+\tau)$, and the item $y(t)$ can be seen as known information for sensor side. So, we just need to handle $\bar{w}(t)$. One have $\dot{\bar{w}}(t)=\dot{w}(t+\tau)=C A \hat{x}(t)+C B(u(t))$ for $t \in\left[\bar{t}_{k}, \bar{t}_{k+1}\right)$ and $\bar{w}\left(\bar{t}_{k}\right)=w\left(\bar{t}_{k}+\tau\right)=w\left(t_{k}\right)=y\left(t_{k}-\tau\right)=y\left(\bar{t}_{k}\right)$, so $\bar{w}(t)$ can be seen as known item when designing event-triggered condition just by setting the following model at the sensor side

$$
f:\left\{\begin{array}{l}
\dot{\bar{w}}(t)=C A \hat{x}(t)+C B(u(t)), t \in\left[\bar{t}_{k}, \bar{t}_{k+1}\right) \\
\bar{w}\left(\bar{t}_{k}\right)=y\left(\bar{t}_{k}\right)
\end{array}\right.
$$

Remark 2. (Design an event-triggered mechanism like (6)) In (6), we just give the event-triggered mechanism first, and the details about how to design it will be shown later as three steps in the 'proof' part of following Theorem 5.1. According to the three steps in the 'proof' part of following Theorem 5.1, in general, the event-triggered mechanism is designed following this idea: (1) Deign a Lyapunov function for observe system, and give the derivative of it; (2) Find an appropriate storage function larger than the derivative of the Lyapunov function, which is only related to available information of sensor; (3) Design an event-triggered mechanism which guarantees this storage function non-positive. That means the sensor needs to detect the real-time value of this storage function, and once it becomes positive, data transmission should be done to make it non-positive (sampling error $e_{w}(t+\tau)=0$ for $t=\bar{t}_{k}$, so that the condition in $(6)$ becomes $(C e(t))^{\prime} F_{11} C e(t)<0$ for $\left.C e(t) \neq 0\right)$. Actually, the aim is to guarantee the derivative of the Lyapunov function to be negative, however not all of the variables in it are available for sensor, so this appropriate storage function has to be found for sensor's detection, helping to guarantee the derivative of the Lyapunov function to be negative.

Remark 3. The event-triggered mechanism (6) and (7) guarantee $\left[C e(t) ; e_{w}(t+\tau)\right]^{\prime} F\left[C e(t) ; e_{w}(t+\tau)\right] \leq 0$ for all $t \geq 0$ which actually also guarantees

$$
\left[\begin{array}{c}
C e(t-\tau) \\
e_{w}(t)
\end{array}\right]^{\prime} F\left[\begin{array}{c}
C e(t-\tau) \\
e_{w}(t)
\end{array}\right] \leq 0
$$

for all $t \geq \tau$ just with a variable substitution, which is an important condition further guaranteeing the decrease of the Lyapunov function in the sequel.

For the designed observer (3) and (4) and the system (2), consider the following Lyapunov function: 


$$
\begin{aligned}
V(e(t))= & {\left[\begin{array}{c}
e(t) \\
\int_{t-\tau}^{t} e(s) d s
\end{array}\right]^{\prime}\left[\begin{array}{cc}
P & Q \\
Q^{\prime} & T
\end{array}\right]\left[\begin{array}{c}
e(t) \\
\int_{t-\tau}^{t} e(s) d s
\end{array}\right]+\int_{t-\tau}^{t} e^{\prime}(s) S e(s) d s } \\
& +\int_{t-\tau}^{t}(\tau-t+s) \dot{e}^{\prime}(s) \operatorname{Re}(s) d s+e_{w}^{\prime}(t) P_{w} e_{w}(t)
\end{aligned}
$$

with $\left[\begin{array}{cc}P & Q \\ Q^{\prime} & T\end{array}\right]>0, S>0, R>0$ and $P_{w}>0$. Thus, in the following Theorem 5.1 and its proof, we will show how to guarantee the decrease of the Lyapunov function (10) by designing the event-triggered mechanism as (6).

Theorem 5.1. Under the proposed observer (3) and (4) for system (2), the decrease of Lyapunov function (10) is guaranteed if there exist positive parameter $\tau>0$, matrices $M \in \mathbb{R}^{n \times(4 n+p)}, F \in \mathbb{R}^{2 p \times 2 p}$ satisfying (7) and positive matrices $S \in \mathbb{R}^{n \times n}, R \in$ $\mathbb{R}^{n \times n}, P_{w} \in \mathbb{R}^{p \times p},\left[\begin{array}{cc}P & Q \\ Q^{\prime} & T\end{array}\right] \in \mathbb{R}^{2 n \times 2 n}$ with $P, Q, T \in \mathbb{R}^{n \times n}$ such that the following condition is satisfied:

$$
\Phi(\tau)+M^{\prime} D+D^{\prime} M-G^{\prime} F G<0 .
$$

where

$$
\begin{aligned}
& D=\left[\begin{array}{lllll}
A & -L C & 0 & -I_{n} & L
\end{array}\right] \\
& G=\left[\begin{array}{ccccc}
0 & C & 0 & 0 & 0 \\
0 & 0 & 0 & 0 & I_{p}
\end{array}\right] \\
& \Phi(\tau)=H_{e}\left(M_{1}^{\prime} P M_{4}+M_{1}^{\prime} Q M_{1}-M_{1}^{\prime} Q M_{2}+M_{3}^{\prime} Q^{\prime} M_{4}+M_{3}^{\prime} T M_{1}-M_{3}^{\prime} T M_{2}\right) \\
& +M_{1}^{\prime} S M_{1}-M_{2}^{\prime} S M_{2}+M_{4}^{\prime} \tau R M_{4}+\frac{-1}{\tau}\left(M_{1}-M_{2}\right)^{\prime} R\left(M_{1}-M_{2}\right) \\
& +\frac{-3}{\tau}\left(M_{1}+M_{2}-\frac{2}{\tau} M_{3}\right)^{\prime} R\left(M_{1}+M_{2}-\frac{2}{\tau} M_{3}\right)+H e\left(M_{5}^{\prime} P_{w} C A M_{2}\right)
\end{aligned}
$$

with

$$
\begin{aligned}
M_{1} & =\left[I_{n}, 0,0,0,0\right] \\
M_{2} & =\left[0, I_{n}, 0,0,0\right] \\
M_{3} & =\left[0,0, I_{n}, 0,0\right] \\
M_{4} & =\left[0,0,0, I_{n}, 0\right] \\
M_{5} & =\left[0,0,0,0, I_{p}\right]
\end{aligned}
$$


Proof. (1) Considering the Lyapunov function (10), one can easily see that $V\left(t_{k}\right)<$ $V^{-}\left(t_{k}\right)$, thus we only need to prove that $\dot{V}(t)<0, t \in\left[t_{k}, t_{k+1}\right)$ in the following part. For $t \in\left[t_{k}, t_{k+1}\right)$, according to the Wirtinger-based inequality shown in Lemma 2.1, the derivative of the Lyapunov function is as follow:

$$
\begin{aligned}
\dot{V}(e(t))= & 2\left[\begin{array}{c}
e(t) \\
\int_{t-\tau}^{t} e(s) d s
\end{array}\right]^{\prime}\left[\begin{array}{cc}
P & Q \\
Q^{\prime} & T
\end{array}\right]\left[\begin{array}{c}
\dot{e}(t) \\
e(t)-e(t-\tau)
\end{array}\right]+e^{\prime}(t) S e(t) \\
& -e^{\prime}(t-\tau) S e(t-\tau)+\tau \dot{e}^{\prime}(t) R \dot{e}(t)+\int_{t-\tau}^{t}-\dot{e}^{\prime}(s) R \dot{e}(s) d s+2 e_{w}^{\prime}(t) P_{w} \dot{e}_{w}(t) \\
\leq & 2\left[\begin{array}{c}
e(t) \\
\int_{t-\tau}^{t} e(s) d s
\end{array}\right]^{\prime}\left[\begin{array}{cc}
P & Q \\
Q^{\prime} & T
\end{array}\right]\left[\begin{array}{c}
\dot{e}(t) \\
e(t)-e(t-\tau)
\end{array}\right]+e^{\prime}(t) S e(t) \\
& -e^{\prime}(t-\tau) S e(t-\tau)+\tau \dot{e}^{\prime}(t) R \dot{e}(t)+\frac{-1}{\tau}\left(\int_{t-\tau}^{t} \dot{e}(s) d s\right)^{\prime} R\left(\int_{t-\tau}^{t} \dot{e}(s) d s\right) \\
& +\frac{-3}{\tau}\left(\int_{t-\tau}^{t}\left(\frac{\tau+2(s-t)}{\tau}\right) \dot{e}(s) d s\right)^{\prime} R\left(\int_{t-\tau}^{t}\left(\frac{\tau+2(s-t)}{\tau}\right) \dot{e}(s) d s\right) \\
& +2 e_{w}^{\prime}(t) P_{w} C A e(t-\tau) \\
= & 2\left[\begin{array}{cc}
e(t) \\
\int_{t-\tau}^{t} e(s) d s
\end{array}\right]^{\prime}\left[\begin{array}{cc}
P & Q \\
Q^{\prime} & T
\end{array}\right]\left[\begin{array}{c}
\dot{e}(t) \\
e(t)-e(t-\tau)
\end{array}\right]+e^{\prime}(t) S e(t) \\
& -e^{\prime}(t-\tau) S e(t-\tau)+\tau \dot{e}^{\prime}(t) R \dot{e}(t)+\frac{-1}{\tau}(e(t)-e(t-\tau))^{\prime} R(e(t)-e(t-\tau)) \\
& +\frac{-3}{\tau}\left(e(t)+e(t-\tau)-\frac{2}{\tau} \int_{t-\tau}^{t} e(s) d s\right)^{\prime} R\left(e(t)+e(t-\tau)-\frac{2}{\tau} \int_{t-\tau}^{t} e(s) d s\right) \\
& +2 e_{w}^{\prime}(t) P_{w} C A e(t-\tau)
\end{aligned}
$$

Introduce the vector as follow:

$$
\xi(t)=\left[\begin{array}{c}
e(t) \\
e(t-\tau) \\
\int_{t-\tau}^{t} e(s) d s \\
\dot{e}(t) \\
e_{w}(t)
\end{array}\right]
$$

Then, for $t \in\left[t_{k}, t_{k+1}\right)$, one gets

$$
\dot{V}(e(t))=\xi^{\prime}(t) \Phi(\tau) \xi(t)
$$


Here, we need to introduce another relationship. According to (5), one gets

$$
\left[\begin{array}{lllll}
A & -L C & 0 & -I_{n} & L
\end{array}\right] \xi(t)=0
$$

According to the above condition (19), one gets

$$
2 \xi^{\prime}(t) M^{\prime}\left[\begin{array}{lllll}
A & -L C & 0 & -I_{n} & L
\end{array}\right] \xi(t)=0
$$

which leads to

$$
\xi^{\prime}(t) M^{\prime} D \xi(t)+\xi^{\prime}(t) D^{\prime} M \xi(t)=0
$$

with

$$
D=\left[\begin{array}{lllll}
A & -L C & 0 & -I_{n} & L
\end{array}\right]
$$

So, the decrease of Lyapunov function (10) is guaranteed if

$$
\Phi(\tau)+M^{\prime} D+D^{\prime} M<0
$$

(2) Next, we need to find an appropriate storage function larger than the derivative of the Lyapunov function (18), which only related available information of sensor.

The available information of sensor related to vector $\xi(t)$, is as follow:

$$
\xi_{e}(t)=\left[\begin{array}{c}
C e(t-\tau) \\
e_{w}(t)
\end{array}\right]
$$

Thus, we find a storage function like this form $\left[\begin{array}{c}C e(t-\tau) \\ e_{w}(t)\end{array}\right]^{\prime} F\left[\begin{array}{c}C e(t-\tau) \\ e_{w}(t)\end{array}\right]$, which may be larger than the derivative of the Lyapunov function (18) by design the matrix $F$.

(3) Then, we design the event-triggered mechanism like (6) so that the following condition is always satisfied:

$$
\left[\begin{array}{c}
C e(t-\tau) \\
e_{w}(t)
\end{array}\right]^{\prime} F\left[\begin{array}{c}
C e(t-\tau) \\
e_{w}(t)
\end{array}\right] \leq 0
$$


Thus, with (25), the decrease of Lyapunov function (10) is guaranteed if

$$
\Phi(\tau)+M^{\prime} D+D^{\prime} M-G^{\prime} F G<0
$$

with

$$
G=\left[\begin{array}{ccccc}
0 & C & 0 & 0 & 0 \\
0 & 0 & 0 & 0 & I_{p}
\end{array}\right]
$$

Proof is ended here.

\subsection{Exclusion of Zeno behaviour and convergence analysis}

In this part, based on the results shown in the above Section 5.1, we will exclude the Zeno behaviour and then give the convergence analysis.

Since the decrease of Lyapunov function has been proved in Theorem 5.1, one can get that the estimation error is bounded, thus we assume $\|e(t)\| \leq \Lambda$ where $\Lambda \in \mathbb{R}$ and $\Lambda>0$. Since condition $F_{11}<0$ is satisfied, it's eigenvalues are all negative, thus we assume the biggest eigenvalues of $F_{11}$ as $\alpha_{F_{11} \max }$, and it satisfies:

$$
\alpha_{F_{11} \max }<0
$$

In practical application, due to the precision of sensor, the event-triggered condition in (6) only can be detected by sensor when $\|C e(t)\|^{2} \geq \rho$ where $\rho \in \mathbb{R}$ is a very small positive constant depending on the precision of sensor. This condition is actually added for excluding the Zeno behaviour in the sequel. This is actually similar to the literature ( $\mathrm{Li}$ et al. (2019)), in which a parameter $\zeta_{2 y}$ is added in event-triggered condition for the same reason. Thus, the transmission period $\bar{t}_{k+1}-\bar{t}_{k}$ can be guaranteed to be positive: At instant $\bar{t}_{k}$, the event is triggered, which means that $w(t)$ will be updated by real output at instant $\bar{t}_{k}+\tau$, and one has $e_{w}\left(\bar{t}_{k}+\tau\right)=0$, so the inter-execution times $\bar{t}_{k+1}-\bar{t}_{k}$ are bounded by the time it takes for $\left[\begin{array}{c}C e(t) \\ e_{w}(t+\tau)\end{array}\right]^{\prime} F\left[\begin{array}{c}C e(t) \\ e_{w}(t+\tau)\end{array}\right]$ to evolve from negative value (as a result of $\left.F_{11}<0\right)$ to positive value. Thus, we give the following results:

Theorem 5.2. For an arbitrary small positive constant $\rho \in \mathbb{R}$ such that $\|C e(t)\|^{2} \geq \rho$, then there exists a positive inter-event time $\bar{t}_{k+1}-\bar{t}_{k}$ satisfying

$$
\bar{t}_{k+1}-\bar{t}_{k}>T
$$


where

$$
T=\frac{-\Lambda^{2}\left\|F_{12}+F_{21}^{\prime}\right\|\|C A\|\|C\|+\Lambda\|C A\| \sqrt{\Lambda^{2}\left\|F_{12}+F_{21}^{\prime}\right\|^{2}\|C\|^{2}-4\left\|F_{22}\right\| \alpha_{F_{11} \max } \rho}}{2\left\|F_{22}\right\|\|C A\|^{2} \Lambda^{2}}
$$

Proof. Assume $t$ is the current time, and $\bar{t}_{k}$ is the last trigger instant. Now, we need to use the event-triggered mechanism (6) to determine the next trigger instant after $\bar{t}_{k}$. Thus, we assume the inequality in (6) is satisfied at certain time instant $t$, causing the next trigger after instant $\bar{t}_{k}$. The inequality in (6) can be written as

$$
e^{\prime}(t) C^{\prime} F_{11} C e(t)+e_{w}^{\prime}(t+\tau) F_{22} e_{w}(t+\tau)+e^{\prime}(t) C^{\prime}\left(F_{12}+F_{21}^{\prime}\right) e_{w}(t+\tau)>0
$$

According to the definition of $e_{w}(t)$, one can get

$$
\dot{e}_{w}(t)=C A e(t-\tau)
$$

Thus, one obtains

$$
\begin{aligned}
\left\|e_{w}(t+\tau)\right\|^{2} & =\left\|e_{w}\left(\bar{t}_{k}+\tau\right)+\int_{\bar{t}_{k}+\tau}^{t+\tau} C A e(t-\tau) d t\right\|^{2} \\
& =\left\|\int_{\bar{t}_{k}+\tau}^{t+\tau} C A e(t-\tau) d t\right\|^{2} \\
& \leq\left(\int_{\bar{t}_{k}+\tau}^{t+\tau}\|C A e(t-\tau)\| d t\right)^{2} \\
& \leq\left(\int_{\bar{t}_{k}+\tau}^{t+\tau}\|C A\|\|e(t-\tau)\| d t\right)^{2} \\
& \leq\|C A\|^{2} \Lambda^{2}\left(t-\bar{t}_{k}\right)^{2}
\end{aligned}
$$

According to (33), the left hand of (31) can be deduced as

$$
\begin{aligned}
& e^{\prime}(t) C^{\prime} F_{11} C e(t)+e_{w}^{\prime}(t+\tau) F_{22} e_{w}(t+\tau)+e^{\prime}(t) C^{\prime}\left(F_{12}+F_{21}^{\prime}\right) e_{w}(t+\tau) \\
& \leq \alpha_{F_{11} \max } \rho+\left\|F_{22}\right\|\|C A\|^{2} \Lambda^{2}\left(t-\bar{t}_{k}\right)^{2}+\Lambda^{2}\left\|F_{12}+F_{21}^{\prime}\right\|\|C A\|\|C\|\left(t-\bar{t}_{k}\right)
\end{aligned}
$$


Thus, if (31) is satisfied for trigger, then according to (34), the following inequality is also satisfied:

$$
\bar{a}\left(t-\bar{t}_{k}\right)^{2}+\bar{b}\left(t-\bar{t}_{k}\right)+\bar{c}>0
$$

where

$$
\begin{gathered}
\bar{a}=\left\|F_{22}\right\|\|C A\|^{2} \Lambda^{2} \\
\bar{b}=\Lambda^{2}\left\|F_{12}+F_{21}^{\prime}\right\|\|C A\|\|C\| \\
\bar{c}=\alpha_{F_{11} \max } \rho
\end{gathered}
$$

From (36)-(38), one can get

$$
\bar{a}>0 ; \bar{b}>0 ; \bar{c}<0
$$

which means the solution of inequality (35) is an open interval, with two sections on the positive and negative axis respectively. Because of $t>\bar{t}_{k}$, ignore the negative part, the inequality (35) has the following solution:

$$
t-\bar{t}_{k}>T
$$

which means if the condition in (6) is satisfied for time instant $t$, and then lead to the next trigger at time instant $t$ after the last trigger instant $\bar{t}_{k}$ (i.e. $t=\bar{t}_{k+1}$ ), then the above inequality (40) is satisfied, which exclude the zeno behaviour.

Theorem 5.3. With the event-triggered mechanism (6), if all conditions in Theorem 5.1 and 5.2 are satisfied, then the state $\hat{x}$ of the proposed observer (3) and (4) asymptotically converges to a small neighborhood (depending on the $\rho$ ) of the state $x$ of system (2) without Zeno behavior.

Proof. With the above result Theorem 5.2 and the previous Theorem 5.1, one can easily get the Theorem 5.3. 
Remark 4. Actually, it is because of the precision of sensor, which leads to no transmissions when $\|C e(t)\|^{2}<\rho$, that a practical convergence of the observer exists.

Remark 5. Although the delay in this paper has no bounds, but if it is too large, it may lead to insolubility of LMIs (11). Actually, if the delay is too large, we may not find a good enough parameter $L$ to make LMIs solvable. In systems with network constraints, because of the time-delays and sampling, we usually can't find a very good or accurate parameter $L$ by set the poles of $A-L C$. Fortunately, in this paper a prediction (4) is used to compensate for the effect of sampling, which may be useful to find a better $L$. Besides, the compensation for the effect of delay is also important, but it is now not considered in this paper and will be done in the further work. And for the compensation of time-delays, a prediction method mentioned in (Gawthrop \& Wang, 2009a, 2009b) can be used.

\section{Application to a visual servoing mobile cart system}

Refer to literatures (Wang, Tian, \& Christov, 2016; Wang, Tian, \& Vasseur, 2015), the considered example is a mobile cart which moves along a horizontal and straight line segment. The cart is moved through a notched belt powered by an electric motor. This referred mobile cart system can be modeled as

$$
\left\{\begin{array}{l}
\dot{x}(t)=\left[\begin{array}{cc}
0 & 1 \\
0 & -\frac{1}{\tau_{c}}
\end{array}\right] x(t)+\left[\begin{array}{c}
0 \\
\frac{k_{c}}{\tau_{c}}
\end{array}\right] u(t) \\
y(t)=\left[\begin{array}{ll}
1 & 0
\end{array}\right] x(t)
\end{array}\right.
$$

where $x(t)=\left[\begin{array}{ll}p_{x}(t) & v_{x}(t)\end{array}\right]^{\prime}$ is the state which is composed of the cart position $p_{x}(t) \in \mathbb{R}$ and the speed $v_{x}(t) \in \mathbb{R}$. The parameters $\tau_{c}=0.83 \mathrm{~s}, k_{c}=2.9 \mathrm{~m} \cdot \mathrm{s} / \mathrm{V}$ are the system time constant and overall gain respectively. And $y(t)$ is the system output.

\subsection{Simulation results}

The initial value of system state is set as $x_{0}=[10 ; 8]$. The fixed time-delays induced by network transmission can be set as $\tau=0.05 \mathrm{~s}$. Select the poles of $A-L * C$ at $(-3.1974-1.9074)$, one can get $L=[3.9 ; 1.4]$. We set $u(t)=2 \sin (t-0.125 \pi)$. The precision is set as $\rho=10^{-5}$ for both Section 6.1 and 6.2. By using the LMI tools in matlab, one can get the solutions 


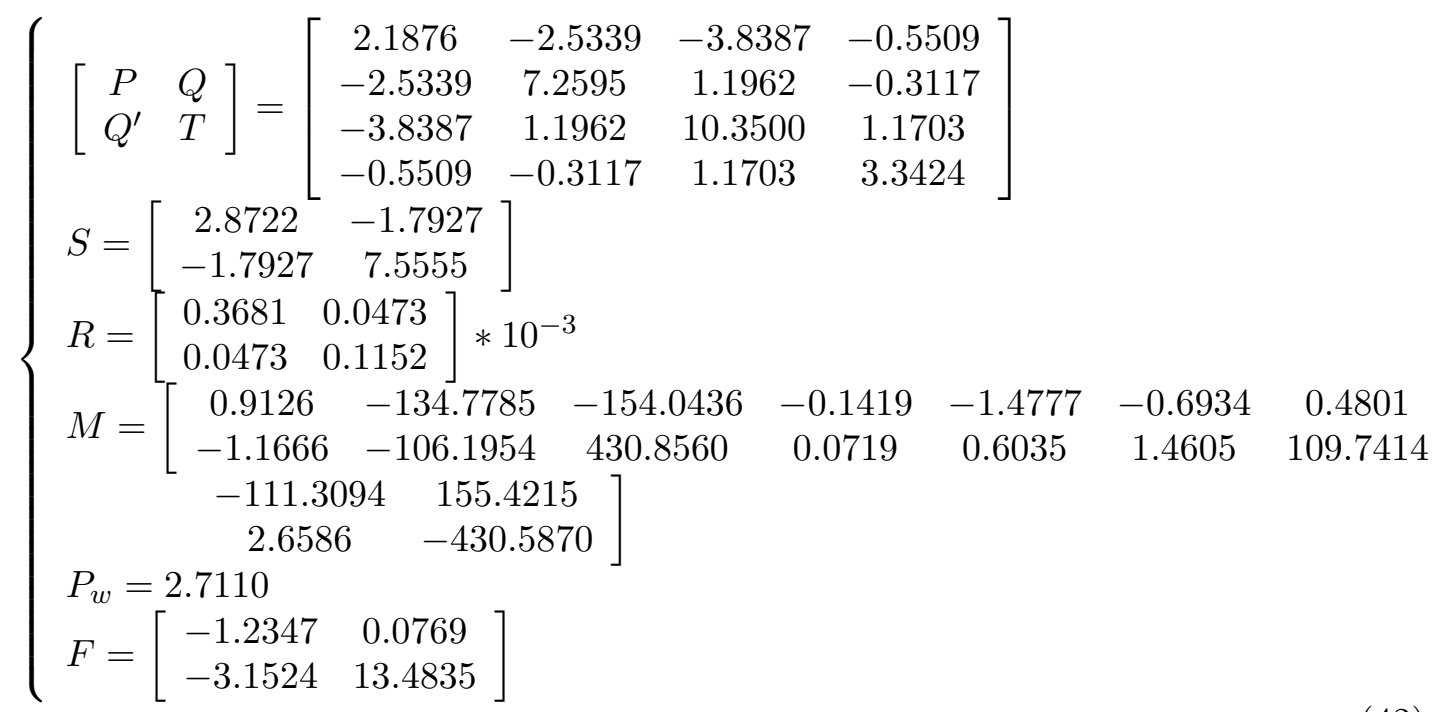

The following figures 2-3 show the estimation results and the trigger signals. For comparison, we also give the results of classical event-triggered mechanism (classical $\mathrm{ETM})$ in form of $\bar{t}_{k+1}=\min \left\{\bar{t} \geq \bar{t}_{k}\right.$, s.t. $\left.\left\|y(t)-y\left(\bar{t}_{k}\right)\right\|^{2} \geq \sigma_{1}\|y(t)\|^{2}+\sigma_{2}\right\}$ with $\sigma_{1}=0.03, \sigma_{2}=0.02$ which is used in literatures Li et al. (2019), and the results of periodic time-triggered mechanism (TTM) with the period setted as $0.9 s$. Seeing from figures 2-3 and table 1, one can find that, compared with the other two methods, the method based on the new event-triggered mechanism proposed in this paper leads to both better estimation performance and larger average transmission period.
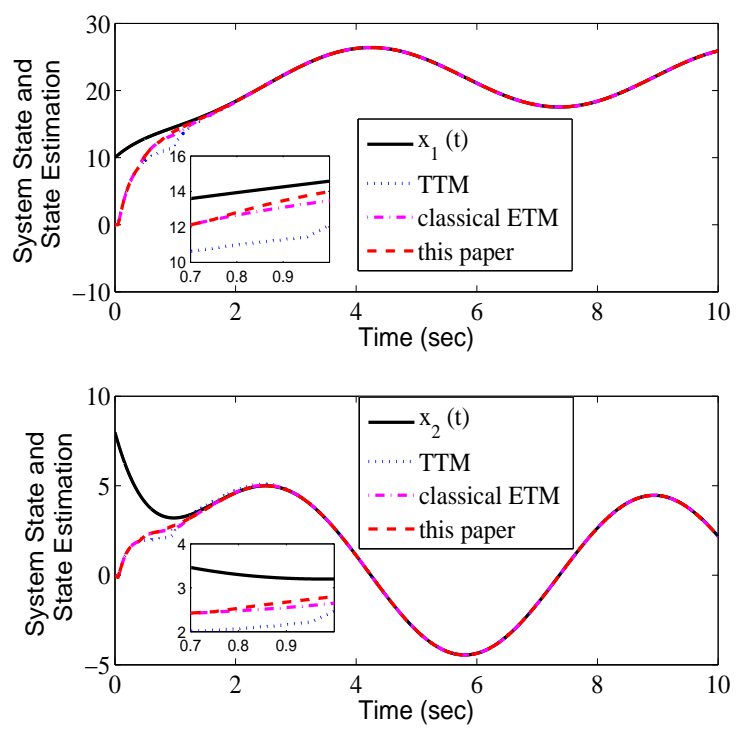

Figure 2. State estimation based on different methods 

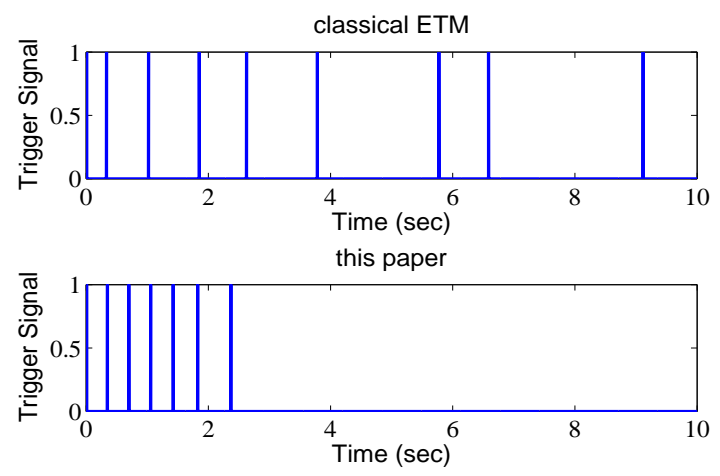

Figure 3. Trigger signal based on different methods

Table 1. Average transmission period in $10 \mathrm{~s}$ based on different methods.

\begin{tabular}{cccc}
\hline Methods & TTM & classical ETM & This paper \\
\hline Average transmission period & 0.9 & 1.11 & 1.43 \\
\hline
\end{tabular}

\subsection{The maximum value of $\tau$ under a certain value of $L$}

Even through in this paper no bound of the time-delays is given on the level of theoretical analysis, it is still necessary in the practical application. Thus, in this part, we would like to find the maximum value of $\tau$ under the certain value of $L$ for the special mobile cart system. We only change the value of the time-delay $\tau$ and keep the other parameters the same as above. Through some calculations, based on the certain parameter $L=[3.9 ; 1.4]$, the maximum value of the time-delay is obtained as $\tau=0.29 \mathrm{~s}$ (if $\tau$ is set as $0.3 \mathrm{~s}$, then the LMIs have no solutions), and in this case one can get the follwing solutions by using the LMI tools in matlab:

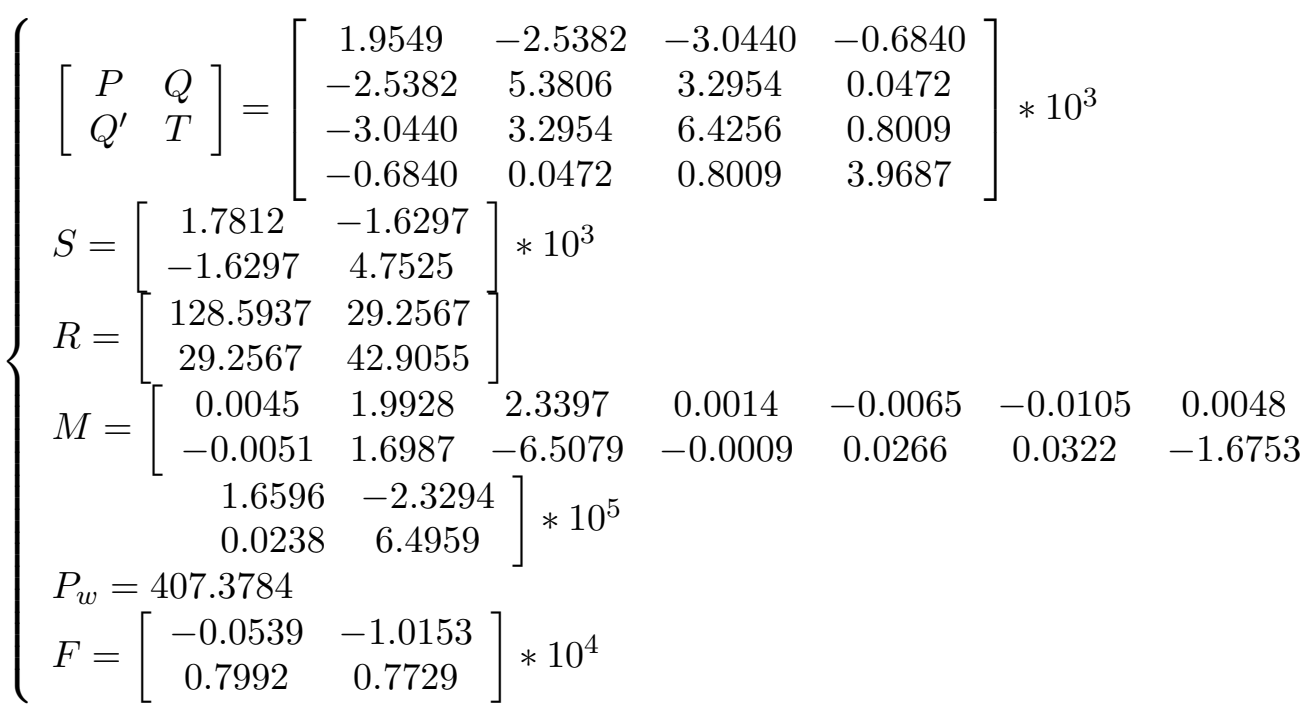


So, with the above calculated parameters, the method can be applied to the mobile cart system with the maximum time-delay $\tau=0.29 \mathrm{~s}$ if we set $L=[3.9 ; 1.4]$, and the corresponding simulation results when $\tau=0.29 \mathrm{~s}$ are as follows:

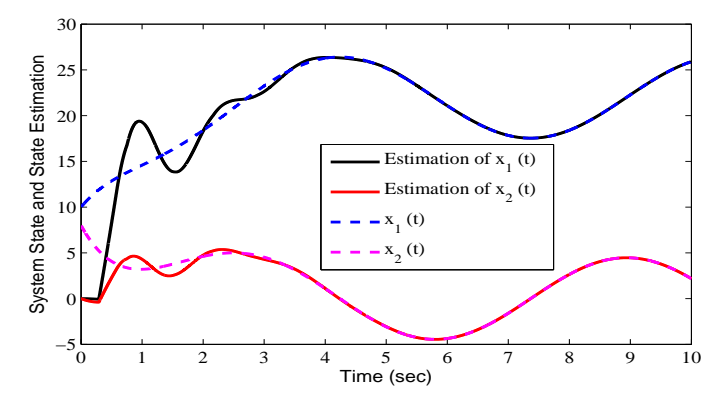

Figure 4. State estimation under $\tau=0.29 \mathrm{~s}$

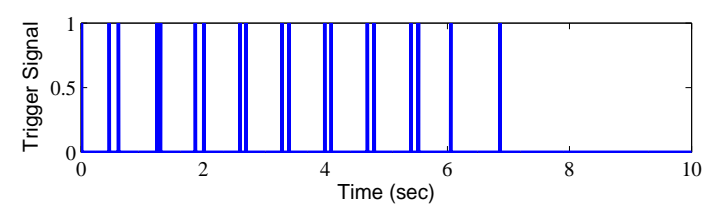

Figure 5. Trigger signal under $\tau=0.29 \mathrm{~s}$

\section{7. conclusion}

An event-triggered observer for linear time-invariant systems is proposed in this paper. Different from the most used classical kind, a new event-triggered mechanism is designed based on an appropriate storage function, which is larger than the derivative of Lyapunov function. This novel event-triggered mechanism is designed to guarantee the storage function non-positive, leading to the decrease of the Lyapunov function, further guaranteeing convergence of the observer system. Compared with a kind of classical event-triggered mechanism and the periodic time-triggered mechanism, the observer based on the new designed event-triggered mechanism brings both better estimation performance and larger average transmission period.

\section{Acknowledgement(s)}

The authors would like to thank both the editor and the reviewers. 


\section{Funding}

This work was partially supported by the National Natural Science Foundation of China under Grants 61773212, the Natural Science Foundation of Jiangsu Province under Grant BK20170094.

\section{References}

Ahmed-Ali, T., Assche, V. V., Massieu, J. F. c., \& Dorleans, P. (2013). Continuous-discrete observer for state affine systems with sampled and delayed measurements. IEEE Transactions on Automatic Control, 58(4), 1085-1091.

Chen, P., \& Li, F. (2018). A survey on recent advances in event-triggered communication and control. Information Sciences, 457-458, 113-125.

Chu, X., \& Li, M. (2018). $H_{\infty}$ observer-based event-triggered sliding mode control for a class of discrete-time nonlinear networked systems with quantizations. Isa Transactions, $79,13-26$.

Dong, H., Wang, Z., Alsaadi, F. E., \& Ahmad, B. (2015). Event-triggered robust distributed state estimation for sensor networks with state-dependent noises. International Journal of General Systems, 44 (2), 254-266.

Etienne, L., \& Gennaro, S. D. (2016). Event-triggered observation of nonlinear lipschitz systems via impulsive observers. IFAC-PapersOnLine, 49(18), 666-671.

Etienne, L., Gennaro, S. D., \& Barbot, J. P. (2017). Periodic event-triggered observation and control for nonlinear lipschitz systems using impulsive observers. International Journal of Robust and Nonlinear Control, 27(4), 1-1.

Gawthrop, P. J., \& Wang, L. (2009a). Constrained intermittent model predictive control. International Journal of Control, 82(6), 1138-1147.

Gawthrop, P. J., \& Wang, L. (2009b). Event-driven intermittent control. International Journal of Control, 82 (12), 2235-2248.

Han, D., Mo, Y., Wu, J., Weerakkody, S., Sinopoli, B., \& Shi, L. (2015). Stochastic eventtriggered sensor schedule for remote state estimation. IEEE Transactions on Automatic Control, 60(10), 2661-2675.

Heemels, W. P. M. H., Sandee, J. H., \& Van Den Bosch, P. P. J. (2008). Analysis of eventdriven controllers for linear systems. International Journal of Control, 81(4), 571-590.

Hetel, L., Fiter, C., Omran, H., Seuret, A., Fridman, E., Richard, J. P., \& Niculescu, S. I. (2017). Recent developments on the stability of systems with aperiodic sampling: An overview. Automatica, 76, 309-335.

Huang, J., Shi, D., \& Chen, T. (2017). Event-triggered state estimation with an energy harvesting sensor. IEEE Transactions on Automatic Control, 62 (9), 4768 - 4775.

Huang, Y., Wang, J., Shi, D., \& Shi, L. (2018). Toward event-triggered extended state observer. IEEE Transactions on Automatic Control, 63(6), 1842-1849.

Leong, A. S., Dey, S., \& Quevedo, D. E. (2017). Sensor scheduling in variance based event triggered estimation with packet drops. IEEE Transactions on Automatic Control, 62(4), 1880-1895.

Li, B., Wang, Z., Ma, L., \& Liu, H. (2019). Observer-based event-triggered control for nonlinear systems with mixed delays and disturbances: The input-to-state stability. IEEE Transactions on Cybernetics, 49(7), 2806-2819.

Molin, A., \& Hirche, S. (2017). Event-triggered state estimation: An iterative algorithm and optimality properties. IEEE Transactions on Automatic Control, 62(11), 5939 - 5946.

Molin, A., Sandberg, H., \& Johansson, K. H. (2016). Consistency-preserving event-triggered estimation in sensor networks. In Ieee conference on decision and control.

Seuret, A., \& Gouaisbaut, F. (2013). Wirtinger-based integral inequality: Application to time-delay systems. Automatica, 49(9), 2860-2866. 
Seuret, A., Prieur, C., Tarbouriech, S., \& Zaccarian, L. (2016). Lq-based event-triggered controller co-design for saturated linear systems. Automatica, 74, 47-54.

Shi, D., Chen, T., \& Ling, S. (2014). Event-triggered maximum likelihood state estimation. Automatica, 50(1), 247-254.

Shoukry, Y., \& Tabuada, P. (2016). Event-triggered state observers for sparse sensor noise/attacks. IEEE Transactions on Automatic Control, 61(8), 2079-2091.

Tanwani, A., Prieur, C., \& Fiacchini, M. (2016). Observer-based feedback stabilization of linear systems with event-triggered sampling and dynamic quantization. Systems and Control Letters, 94, 46-56.

Tarbouriech, S., Seuret, A., Silva, J. M. G. D., \& Sbarbaro, D. (2016). Observer-based eventtriggered control co-design for linear systems. Iet Control Theory and Applications, 10(18), 2466-2473.

Wang, H. P., Tian, Y., \& Christov, N. (2016). Piecewise-continuous observers for linear systems with sampled and delayed output. International Journal of Systems Science, 47(8), 18041815.

Wang, H. P., Tian, Y., \& Vasseur, C. (2015). Piecewise continuous hybrid systems based observer design for linear systems with variable sampling periods and delay output. Signal Processing, 114, 75-84.

Zhang, D., Shi, P., Wang, Q. G., \& Yu, L. (2017). Analysis and synthesis of networked control systems: A survey of recent advances and challenges. Isa Transactions, 66, 376-392.

Zhang, X. M., Han, Q. L., \& Zhang, B. L. (2017). An overview and deep investigation on sampled-data-based event-triggered control and filtering for networked systems. IEEE Transactions on Industrial Informatics, 13(99), 4-16.

Zhang, Z. H., \& Yang, G. H. (2017). Event-triggered fault detection for a class of discrete-time linear systems using interval observers. Isa Transactions, 68, 160-169.

Zheng, X., \& Fang, H. (2016). Recursive state estimation for discrete-time nonlinear systems with event-triggered data transmission, norm-bounded uncertainties and multiple missing measurements. International Journal of Robust and Nonlinear Control, 26(17), n/a-n/a.

Zou, L., Wang, Z., Gao, H., \& Liu, X. (2017). Event-triggered state estimation for complex networks with mixed time delays via sampled data information: The continuous-time case. IEEE Transactions on Cybernetics, 45(12), 2804-2815. 